\title{
Upfront FOLFOXIRI + bevacizumab followed by fluoropyrimidin and bevacizumab maintenance in patients with molecularly unselected metastatic colorectal cancer
}

Alexander Stein ${ }^{1}$, Djordje Atanackovic ${ }^{1}$, Bert Hildebrandt ${ }^{2}$, Patrick Stübs ${ }^{3}$, Wolfram Brugger ${ }^{4}$, Gunnar Hapke ${ }^{5}$, Claus-Christoph Steffens ${ }^{6}$, Gerald Illerhaus ${ }^{7}$, Ernst Bluemner ${ }^{8}$, Jan Stöhlmacher ${ }^{9}$ and Carsten Bokemeyer ${ }^{\star}, 1$

${ }^{1}$ University Medical Centre Hamburg-Eppendorf, Martinistrasse 52, Hamburg 20246, Germany; ${ }^{2}$ Charité Universitätsmedizin Berlin, Campus Charité Mitte, Charitéplatz 1, Berlin 10117, Germany; ${ }^{3}$ University Hospital Magdeburg, Leipziger Strasse 44, Magdeburg 39120, Germany; ${ }^{4}$ Schwarzwald-Baar Klinikum, Klinikstrasse 11, Villingen-Schwenningen 78052, Germany; ${ }^{5}$ Marienkrankenhaus, Alfredstrasse 9, Hamburg 22087, Germany; ${ }^{6}$ MVZ Hämatologie/Onkologie Klinik Dr Hancken, Harsefelder Str. 8, Stade 21680, Germany; ${ }^{7}$ Klinikum Stuttgart, Kriegsbergstraße 60, Stuttgart 70174, Germany; ${ }^{8}$ Ecron Acunova GmbH, Hahnstrasse 70, Frankfurt 60528, Germany and ${ }^{9}$ Tumorgenetik Bonn, Maximilianstrasse 28d, Bonn 53111, Germany

Background: The addition of bevacizumab (BEV) to standard doublet chemotherapy improves outcomes compared with chemotherapy alone in patients with metastatic colorectal cancer (mCRC). The OPAL study examined the effect of BEV+ FOLFOXIRI followed by 5FU/LV and BEV maintenance on progression-free survival (PFS) in patients with previously untreated unresectable $\mathrm{mCRC}$.

Methods: Eligible patients had histologically confirmed mCRC, ECOG performance status $\leqslant 1$ and were $18-70$ years old. Patients received up to 12 cycles of FOLFOXIRI + BEV q2w (induction phase) followed by up to $\leqslant 40$ cycles of 5FU/LV+ BEV q2w (maintenance phase). Median PFS was the primary end point; secondary end points included response, OS, secondary resection rate, safety and prognostic value of pharmacogenetic profiling.

Results: Ninety-seven patients were enrolled. Of these, 90 received study medication and formed the safety population: 64 males; median age 58 (range 28-71) years; ECOG performance status 0/1 in 54\%/46\% patients; and liver only disease in 35 patients. Relative dose intensities were 79-85\% for all four drugs. The incidence of adverse events (AEs) was as previously reported and there were no new safety signals. In total, 87 serious AEs occurred in 39 patients (43\%). Median PFS was 11.1 months (95\% Cl 9.4 12.0) and did thus not meet the primary objective of 12 months. Median OS was 32.2 months (95\% $\mathrm{Cl} 22.6-36.9)$. Fifty-two patients were pharmacogenetically profiled.

Conclusions: FOLFOXIRI + BEV was feasible in this molecularly unselected mCRC patient population, showing a high efficacy in terms of survival, overall response and secondary resection rate. Pharmacogenomic profiling revealed no clinically relevant marker.

Colorectal cancer (CRC) is the second (in women) and third (in men) most commonly diagnosed malignancy and one of the leading causes of cancer deaths (Malvezzi et al, 2014). Around one quarter of patients with CRC present with metastatic disease (mCRC) at the time of diagnosis (synchronous disease), and up to $40 \%$ of patients will develop metastases during the course of their

*Correspondence: Professor C Bokemeyer; E-mail: c.bokemeyer@uke.de

Received 12 April 2015; revised 2 July 2015; accepted 27 July 2015; published online 3 September 2015

(c) 2015 Cancer Research UK. All rights reserved 0007-0920/15 
disease, resulting in the relatively high overall mortality rate associated with CRC.

Several drugs as single agent or in various combinations and sequences are available for $\mathrm{mCRC}$, including fluoropyrimidines (5FU, capecitabine), irinotecan, oxaliplatin, the vascular endothelial growth factor (VEGF) antibody bevacizumab, the epidermal growth factor receptor (EGFR) antibodies cetuximab and panitumumab for RAS wild-type patients, the VEGF receptors 1 and 2 fusion protein aflibercept and the multi target tyrosine kinase inhibitor regorafenib (Van Cutsem et al, 2014). Notably, median overall survival (OS) can now be as long as 30 months in molecularly or clinically selected patients with current treatment regimen (Douillard et al, 2013; Heinemann et al, 2014; Loupakis et al, 2014; Venook et al, 2014).

Besides chemotherapy doublets in combination with an antibody, triplet chemotherapy has been established combining fluoropyrimidines, oxaliplatin and irinotecan in first-line mCRC (Souglakos et al, 2006; Falcone et al, 2007; Ychou et al, 2008). The FOLFOXIRI regimen demonstrated a superior overall response rate (ORR), secondary resection rate, progression-free survival (PFS) and OS compared with FOLFIRI (Falcone et al, 2007).

Intensification of first-line therapies with a four-drug combination, including a chemotherapy triplet (fluoropyrimidine, oxaliplatin, irinotecan) and a monoclonal antibody, seems to be a promising approach in terms of increasing the number of patients potentially amenable for secondary local ablation and/or inducing a relevant, long-lasting tumour response. Thus the exploratory phase II OPAL study was performed applying up to 6 months of induction treatment with FOLFOXIRI and bevacizumab followed by up to 18 months of maintenance treatment with 5FU/LV and bevacizumab.

To evaluate the prognostic value of single-nucleotide polymorphisms (SNPs) in the VEGF pathway, an exploratory translational project was performed in a subset of 52 patients (Gerger et al, 2011; Hansen et al, 2012; Loupakis et al, 2013).

\section{PATIENTS AND METHODS}

Patient eligibility. Patients were eligible for inclusion if they were aged between 18 and 70 years; had a histologically confirmed mCRC with at least one radiographically measurable lesion, which was not primarily resectable; an Eastern Cooperative Oncology Group Performance Status (ECOG PS) $\leqslant 1$; and adequate hepatic, renal and bone marrow function. Patients were ineligible if they had a history of previous chemotherapy for mCRC or had uncontrolled severe organ or metabolic dysfunction. The trial was approved by the institutional review board and the competent authority (Paul Ehrlich Institut) and registered (NCT00940303/ EudraCT number: 2008-001180-11). All patients provided written informed consent before study entry.

Study design and treatment. This was an open-label, single arm, multicentre phase II trial evaluating the efficacy and tolerability of FOLFOXIRI and bevacizumab in first-line mCRC and correlating efficacy with pharmacogenomic data in a subgroup of patients. Safety data were assessed after 20 patients had been treated for at least two cycles and again upon completion of induction treatment.

Induction treatment with FOLFOXIRI and bevacizumab was administered with oxaliplatin at a dose of $85 \mathrm{mg} \mathrm{m}^{-2}$ IV over $2 \mathrm{~h}$ (day 1), irinotecan at a dose of $165 \mathrm{mg} \mathrm{m}^{-2} \mathrm{IV}$ over $1 \mathrm{~h}$ (day 1 ), $\mathrm{LV}$ at a dose of $200 \mathrm{mg} \mathrm{m}^{-2} \mathrm{IV}$ over $2 \mathrm{~h}$ (day 1), 5FU at a dose of $3200 \mathrm{mg} \mathrm{m}^{-2} \mathrm{IV}$ over $48 \mathrm{~h}$ (days $1-3$ ) and bevacizumab at a dose of $5 \mathrm{mg} \mathrm{kg}^{-1}$ IV over 30-90 min (day 1) in a biweekly schedule for a maximum of 12 cycles followed by an obligatory maintenance with 5FU/LV and bevacizumab (same dose as used in the induction phase with or without dose reductions) for up to
2 years (Masi et al, 2010; Loupakis et al, 2014). Premature entry into maintenance treatment phase was possible after completing at least six cycle of induction treatment in case of repeated treatment delays or-at the discretion of the investigator-due to toxicity reasons leading to permanent discontinuation of oxaliplatin and irinotecan. Treatment was applied until the occurrence of secondary resection, progressive disease (PD), unacceptable toxicity or withdrawal of consent. Radiographic assessment of response was carried out every 8 weeks until PD or withdrawal for any reason.

Translational pharmacogenomic analyses. Pharmacogenomic analyses of SNPs of the VEGF pathway (for VEGF-A, VEGFR 1-3, PDGFR beta, HIF 1 alpha, Neuropilin) and determination of KRAS status (codons 12, 13 and 61) were performed in a subgroup patients after obtaining separate consent. The main focus of pharmacogenetic analysis was the prediction of the added effect of bevacizumab. Therefore promising candidate SNPs were chosen from recent analyses (Lambrechts et al, 2013). DNA was extracted from white blood cells for SNP analysis and from paraffin-embedded tumour tissue for KRAS analysis. For extraction, QIAamp DNA Mini Kit (Qiagen, Hilden, Germany) was used. Tumour samples were macrodissected before extraction to ensure sufficient tumour DNA within the analyzed sample. Primers for SNP analysis were applied according to the work summarised by Lambrechts et al (2013). Results were correlated with PFS and OS. Because of ethical and legal requirements, the extracted DNA had to be destroyed after the preliminary planned analyses were performed. Therefore, extended RAS (KRAS exon 4 and NRAS exons 2-4) and BRAF mutational testing could not be performed.

Dose adjustments. A new treatment cycle was scheduled if the neutrophil count was $\geqslant 1500 \mathrm{~mm}^{-3}$, the platelet count was $\geqslant 75000 \mathrm{~mm}^{-3}$, if treatment-related diarrhoea and/or abdominal cramps were fully resolved to baseline or grade 0 and no loperamide had been administered during the last $24 \mathrm{~h}$ and all relevant non-haematological toxic effects were grade $\leqslant 1$ (NCI CTC AE v 3.0). Dose reductions were based on the toxicity in the preceding cycle and were performed in $25 \%$ steps for $5 \mathrm{FU}$, irinotecan and oxaliplatin. Treatment was held for grade 3 nonhaematological adverse events (AEs; excluding alopecia, nausea or vomiting), until resolution to grade $\leqslant 1$, and resumed at a $25 \%$ reduction of doses of all three drugs, and discontinued for grade 4 non-haematological adverse. In case of a drug-specific AE, for example, peripheral neuropathy for oxaliplatin solely, the suspected drug was reduced or discontinued.

Study evaluations. Pretreatment evaluation included a complete medical history, physical examination, routine haematology, biochemistry and urine analyses and computed tomography (CT) scans of the chest and abdomen. Haematological (including platelet and differential) analyses, serum chemistry and urine dipstick were obtained at day 1 in each cycle. Subjective symptoms, physical examination results, vital signs (including blood pressure), performance status and all adverse reactions were recorded before each treatment cycle according to NCI CTC AE v 3.0. CT scans were performed every 8 weeks (four cycles) during treatment and afterwards every 12 weeks to assess disease status. ORR and PFS were evaluated according to Response Evaluation Criteria in Solid Tumours (RECIST; Therasse et al, 2000). Archival tissue and $5 \mathrm{ml}$ blood at baseline were obtained for pharmacogenomic analyses in patients giving separate consent.

Statistical methods. The primary end point was median PFS according to RECIST measured from the start of treatment. The sample size calculation was based on an assumed median PFS of 12 months for FOLFOXIRI and bevacizumab followed by a bevacizumab-based maintenance therapy to show an improvement 
of median PFS in comparison to a supposed true value of 8.9 months of a three-drug regimen in molecularly unselected firstline mCRC patients. This resulted in a sample size of 87 patients (one-sided alpha-level of $5 \%$, power $80 \%$, drop-out rate $10 \%$ and follow-up 36 month). An interim analysis was performed 18 months after inclusion of the first patient. As this was an exploratory study, no adjustment of $P$-values or formal statistical interim analysis methodology were applied. Baseline patient characteristics, response and toxic effects were described using summary statistics. The Kaplan-Meier-method was used to analyse the primary end point and censored event times. 95\% Confidence intervals (CIs) were given for all the calculated estimates.

\section{RESULTS}

Patients' characteristics. Between July 2009 and July 2011, a total of 97 patients were screened at 16 German study sites. Seven patients were not included owing to violation of selection criteria. Baseline characteristics are summarised in Table 1. Twenty-six female and 64 male patients with median age of 58 years (range 28-71 years) and ECOG PS score of $0 / 1$ in 54\%/46\%, respectively, were analysed. Site of primary tumour was rectal in 35 (39\%) patients. Prior adjuvant treatment was administered in 7 patients (8\%), single agent fluoropyrimidine in 5 and fluoropyrimidine and oxaliplatin in 2 patients, respectively.

Study treatment. A total of 1342 cycles was administered. Patients received a median of 12 cycles of bevacizumab (range 1-52) and 11.5 cycles of FOLFOXIRI (range 1-12). Relative dose intensities (mean \pm s.d.) were $85 \pm 14 \%$ for bevacizumab, $85 \pm 16 \%$ for oxaliplatin, $83 \pm 16 \%$ for irinotecan and $79 \pm 15 \%$ for $5 \mathrm{FU}$. The mean study duration was 23 months, including a mean treatment duration of 8 months and a mean follow-up duration of 15 months thereafter.

Overall, 29 patients (32\%) had to be dose reduced. Study drug(s) were temporarily withdrawn in 43 (48\%) patients. Reason for discontinuation of study treatment were PD in 36 (41\%), toxicity in $14(16 \%)$, complete remission in $5(6 \%)$, withdrawal of consent in 1 , death in 1 and other in 31 patients (mainly patient request or secondary resection).

Colony-stimulating factors were administered in 13 patients (14\%).

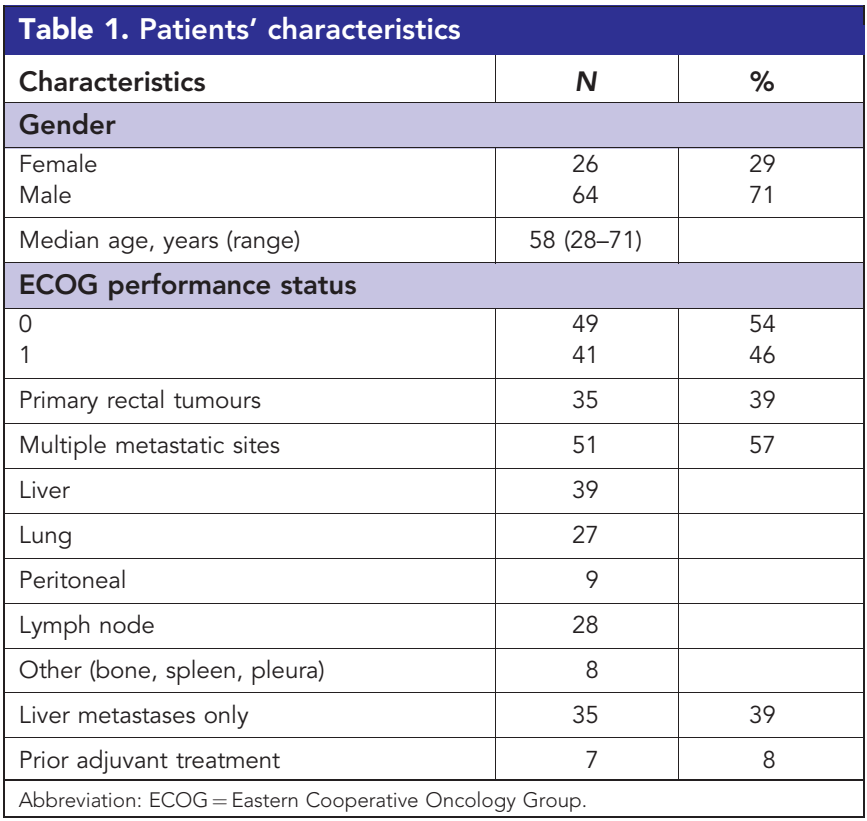

Overall, 64 patients (74\%) received second-line treatment. Patients progressing during maintenance with 5FU/LV and bevacizumab $(n=19)$ were reinduced with oxaliplatin $(n=6)$, irinotecan $(n=12)$ or both $(n=1)$ in combination with bevacizumab $(n=8)$ or EGFR antibodies $(n=3)$. Further salvage treatment contained cetuximab in 15 patients and panitumumab in 18 patients.

Efficacy. The efficacy results were determined in all patients enrolled in the OPAL trial. The intent-to-treat population contained 90 and the per protocol population 87 patients. The group of patients receiving at least one treatment $(n=90)$ formed the safety population.

Results are summarised in Table 2. ORR was $64 \%$ with 7 complete (8\%) and 51 partial responses (57\%). Stable disease as best response was achieved in 20 patients (22\%), resulting in a disease stabilisation rate of $86.7 \%$. The median PFS was 11.1 months (95\% CI (9.4-12.0); Figure 1) and median OS was 32.2 months (95\% CI (22.6-36.9); Figure 2; Kaplan-Meier estimates). The primary objective assuming a median PFS of 12 months was not met.

During the whole study period, 24 (27\%) patients received secondary resections, with 16 patients being curatively resected, leading to an $\mathrm{R} 0$ resection rate of $18 \%$. The resection rate in patients with liver-limited disease $(n=35)$ was similar ( 9 patients, $26 \%)$. Median OS in patients with secondary resection was 36.9 months (95\% CI (32.0-36.9)) and 22.6 months (95\% CI $(18.5-32.6))$ in patients without secondary resection $(n=66)$. After $\mathrm{R} 0$ resection, disease recurred within a median of 4.8 months in 6 patients $(37.5 \%)$. The remaining 10 patients stayed disease free during the follow-up period.

Toxicity. Treatment was generally well tolerated in an outpatient setting (Figure 3). AEs are summarised in Table 3. The most frequent AEs of CTC grade 3/4/5 were leucopenia and neutropenia $(26 \%)$, diarrhoea $(11 \%)$, nausea $(9 \%)$, vomiting (8\%) and venous thromboembolism (6\% of patients).

In total, 87 serious AEs occurred in 39 patients (43\%). Twentyfour of the 87 SAEs (28\%) were assessed by the investigators as related to study medication. Besides 2 patients, suffering from portal vein thrombosis and pulmonary embolism, respectively, all patients recovered from their related SAEs at the end of the study.

A total of 44 patients died during participation in the study, 1 patient during the treatment period and 43 patients during the survival follow-up. Two of these fatal cases were reported as AEs (hepatic failure and infected neoplasm). The investigators assessed both events as unrelated to study treatment.

Pharmacogenetically and KRAS defined subgroups. With regard to the optional participation in the translational part of the trial,

Table 2. Efficacy according to RECIST 1.0 and resection rate

\begin{tabular}{|l|c|c|}
\hline Efficacy & N & $\%$ \\
\hline Response rate & 7 & 8 \\
\hline Complete response & 51 & 57 \\
Partial response & 20 & 22 \\
Stable disease & 7 & 8 \\
Progressive disease or death & 5 & 6 \\
No data on tumour status & 24 & 27 \\
\hline Secondary resection rate & 16 & 18 \\
\hline RO resection rate & 11.1 & $9.4-12.0$ \\
\hline \multicolumn{2}{|l|}{32.2} & $22.6-36.9$ \\
\hline PFS, months & survival; PFS = progression-free \\
\hline OS, months & \\
\hline Abbreviations: Cl=confidence interval; OS=overall & \\
survival. &
\end{tabular}




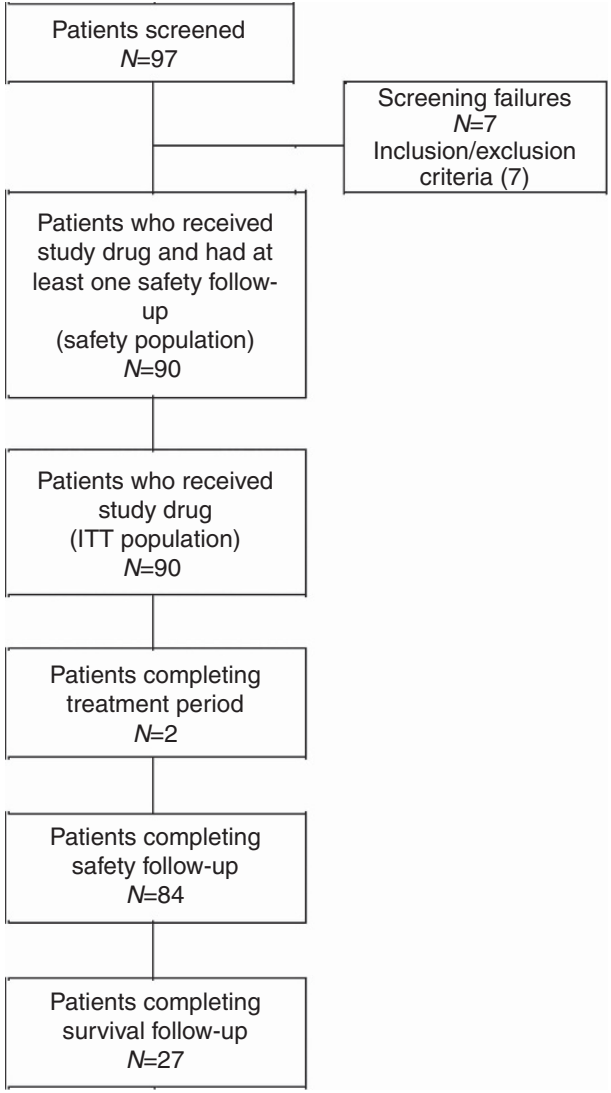

Figure 1. Consort diagram of patient disposition.

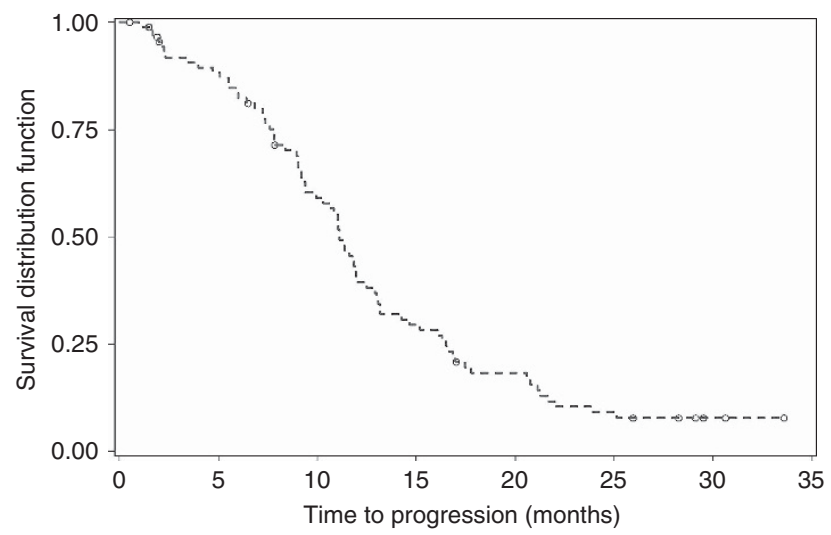

Figure 2. Progression-free survival.

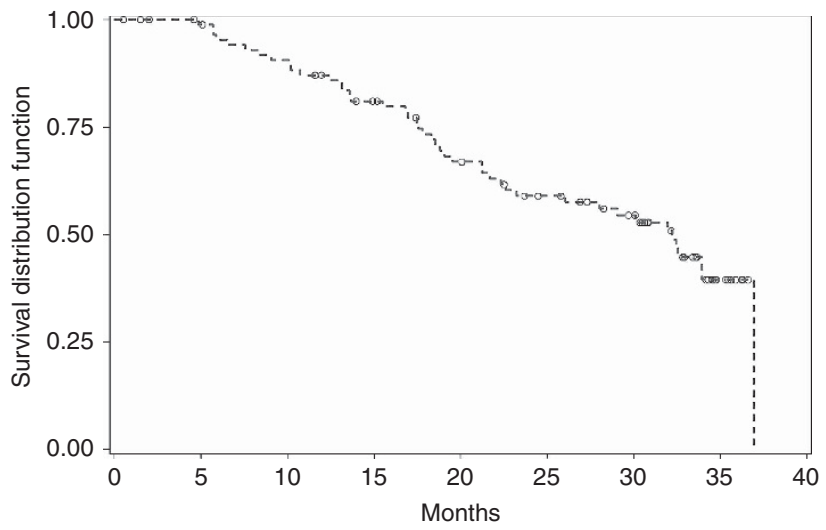

Figure 3. Overall survival.
Table 3. Grade $3 / 4$ toxicity according to National Cancer Institute Common Toxicity Criteria Version 3

Patients with grade $3 / 4$ event, $n(\%)$

\begin{tabular}{|l|c|c|c|}
\hline & $\begin{array}{c}\text { Patients } \\
\text { with all } \\
\text { grade } \\
\text { events, } \\
\text { Adverse event }\end{array}$ & $\begin{array}{c}\text { Induction } \\
\text { phase }\end{array}$ & $\begin{array}{c}\text { Maintenance } \\
\text { phase }\end{array}$ \\
\hline Leucopenia/neutropenia & $43(48)$ & $23(26)$ & 0 \\
\hline Febrile neutropenia & $1(1)$ & $1(1)$ & 0 \\
\hline Anaemia & $14(16)$ & $1(1)$ & 0 \\
\hline Alopecia & $17(19)$ & NA & NA \\
\hline Fatigue & $35(39)$ & $3(3)$ & 0 \\
\hline Stomatitis & $33(37)$ & $4(4)$ & $1(1)$ \\
\hline Diarrhoea & $65(72)$ & $10(11)$ & 0 \\
\hline Nausea & $59(66)$ & $8(9)$ & 0 \\
\hline Vomiting & $30(33)$ & $7(8)$ & 0 \\
\hline Abdominal pain & $29(32)$ & $4(4)$ & $1(1)$ \\
\hline Neurotoxicity & $69(77)$ & $5(6)$ & $1(1)$ \\
\hline Hypertension & $19(21)$ & $3(3)$ & 0 \\
\hline Venous thromboembolism & $14(16)$ & $4(4)$ & $1(1)$ \\
\hline Abbreviation: NA=not applicable. & & & \\
\hline
\end{tabular}

blood and tissue was available from 52 patients (58\% of the whole study population). Despite one failure in KRAS analysis owing to insufficient tumour content in the tissue block, all analyses could be performed as planned and are displayed in Supplementary Table S1. For VEGFR2_FLK_KDR_305_C_T, the median OS was 18.7 months (95\% CI: $16.8-32.2$ months) for polymorphism CT and 30.1 months (95\% CI: 19.5-36.9 months) for polymorphism TT $(P=0.0380)$. The other SNPs were not associated with OS in univariate analyses.

Patients with KRAS-mutated tumours (codon 12, 13 or 61, $n=26)$ had a significantly shorter OS fo 21.7 months compared with 36.9 months $(P=0.027)$ in patients with KRAS wild type $(n=25)$. Because of ethical and legal requirements, the extracted DNA had to be destroyed after the preliminary planned analyses were performed. Therefore, an extended KRAS and NRAS analysis could not be performed in these patients. Importance of extended RAS and BRAF mutational analyses for mCRC was not known by the time of study design.

\section{DISCUSSION}

Four-drug regimens with $5 \mathrm{FU} / \mathrm{LV}$, irinotecan, oxaliplatin and a monoclonal antibody have been evaluated in single-arm phase 2 trials and have shown an ORR ranging from $77 \%$ to $89 \%$ and a PFS of 9.5-13.1 months (Masi et al, 2010; Assenat et al, 2011; Fornaro et al, 2013). Recently, the results of the phase 3 randomised TRIBE trial comparing FOLFIRI and bevacizumab with or without oxaliplatin were reported, showing a significantly increased PFS of 12.1 vs 9.7 months (HR 0.75 ; 95\% CI $(0.62-0.90) ; P=0.003)$ and OS with 29.8 vs 25.8 months (HR 0.80 ; 95\% CI (0.65-0.98); $P=0.03$ ), in favour of FOLFOXIRI and bevacizumab (Loupakis et al, 2014; Loupakis et al, 2015). ORR with FOLFOXIRI and bevacizumab was $65 \%$.

Despite the common differences between phase 2 and 3 trials, particularly in terms of patient selection, the efficacy results of FOLFOXIRI and bevacizumab in the OPAL study mirror the results of the recently reported TRIBE trial. Notably, the OPAL trial did not seem to have a better patient population, with regard 
to an ECOG PS score of 0 in only $54 \%$ of patients in OPAL compared with $90 \%$ in TRIBE (Loupakis et al, 2014). The secondary R0 resection rates in TRIBE and OPAL were similar with $15 \%$ and $18 \%$, respectively. The recently reported OLIVIA trial compared FOLFOX and bevacizumab with or without irinotecan in 80 patients with unresectable liver-limited mCRC showing an ORR of $81 \%$ and a R0 resection rate of $49 \%$ for FOLFOXIRI and bevacizumab, clearly showing the high potential of this regimen to induce secondary resectability in a selected patient population (Gruenberger et al, 2015).

Despite the relatively high-dose intensity of $79-85 \%$, the treatment was well tolerated. The noted toxicities are within the same range as reported for the other three-drug first-line regimen, thus further underlining the feasibility of this intensive first-line approach (Falcone et al, 2007; Douillard et al, 2010; Heinemann et al, 2014).

Interestingly, the particular benefit of the four-drug combination in the KRAS wild-type subgroup as shown in the TRIBE trial (median OS of 41 months) could similarly be shown in the OPAL study showing an OS of 36.9 months in this subgroup of patients, thus further enforcing the discussion about choice of first-line regimen. This may either indicate a prognostic/predictive effect for the agents used or reflect the additional treatment option with EGFR antibodies as salvage therapy for (K)RAS wild-type patients in contrast to the RAS mutant subgroup. However, interpretation of KRAS results is limited by the rather small number of patients with only $57 \%$ of samples available only for KRAS codon 12,13 or 61 analyses. As previously stated, samples had to be destroyed after prespecified analyses. Obtaining extended patient approval for determination of extended RAS and BRAF status was not possible, owing to the recruitment period until 2011.

The pharmacogenomic analyses of the VEGF pathway identified no clinically relevant SNP predictive for treatment with bevacizumab in combination with FOLFOXIRI. The VEGFR2 FLK_KDR_305 SNP subgroup did show a median OS of 18.7 months for the CT SNP $(n=19)$ and 30.1 for the TT SNP $(n=22)$ $(P=0.038)$ in univariate analyses. The exact mechanism of this SNP has not yet been identified. However, as the VEGF receptor represents a natural target for VEGF, its malfunction may relevantly add to the inhibitory effect of bevacizumab on VEGF. With regard to the complexity of the mechanisms of angiogenesis and the potential influence of different chemotherapeutic agents, the prediction of survival by a single SNP seems to be highly unlikely.

These exploratory pharmacogenomic analyses were not adjusted for multiple testing. Moreover, these analyses were performed only in a subgroup of study patients and in a non-randomised setting without a bevacizumab-free control arm.

\section{CONCLUSION}

FOLFOXIRI and bevacizumab has proven to be a feasible and highly efficacious treatment in molecularly unselected first-line mCRC patients enabling a high rate of secondary resection and clearly indicating that the upfront use of several active agents does not compromise survival owing to the lack of available agents for further lines. By applying the selection criteria of OPAL (or TRIBE) study (e.g., age up to 70 years and ECOG PS score 0 or 1 ), the tolerability of the regimen is ensured.

\section{ACKNOWLEDGEMENTS}

We thank the patients and their families, the study teams at Roche and Ecron Acunova and the investigators and study coordinators of all participating sites. The trial was funded and sponsored by Roche. Trial medication (bevacizumab) was supplied by Roche.

\section{CONFLICT OF INTEREST}

AS has received honoraria from Roche, Sanofi, Bayer, Amgen and Merck Serono and research funding from Roche and Sanofi. CB has received honoraria from Merck Serono, Roche, Sanofi and Bayer and research funding from Roche and Merck Serono. EB is an employee of Ecron Acunova, the contract research organisation that was in charge of analysing the study results. JS has received honoraria by Roche, Sanofi, Lilly, Amgen, Bayer, Merck-Serono, Pfizer and Abbott and research funding from Lilly, Roche and Amgen. CCS has received honoraria from Roche, Amgen and Sanofi. BH has received honoraria from Amgen, Bayer, Merck Serono, Roche and Sanofi and research funding from Roche. The remaining authors declare no conflict of interest.

\section{REFERENCES}

Assenat E, Desseigne F, Thezenas S, Viret F, Mineur L, Kramar A, Samalin E, Portales F, Bibeau F, Crapez-Lopez E, Bleuse JP, Ychou M (2011) Cetuximab plus FOLFIRINOX (ERBIRINOX) as first-line treatment for unresectable metastatic colorectal cancer: a phase II trial. Oncologist 16(11): 1557-1564.

Douillard JY, Oliner KS, Siena S, Tabernero J, Burkes R, Barugel M, Humblet Y, Bodoky G, Cunningham D, Jassem J, Rivera F, Kocakova I, Ruff P, Blasinska-Morawiec M, Smakal M, Canon JL, Rother M, Williams R, Rong A, Wiezorek J, Sidhu R, Patterson SD (2013) Panitumumab-FOLFOX4 treatment and RAS mutations in colorectal cancer. N Engl J Med 369(11): 1023-1034.

Douillard JY, Siena S, Cassidy J, Tabernero J, Burkes R, Barugel M, Humblet Y, Bodoky G, Cunningham D, Jassem J, Rivera F, Kocakova I, Ruff P, Blasinska-Morawiec M, Smakal M, Canon JL, Rother M, Oliner KS, Wolf M, Gansert J (2010) Randomized, phase III trial of panitumumab with infusional fluorouracil, leucovorin, and oxaliplatin (FOLFOX4) versus FOLFOX4 alone as first-line treatment in patients with previously untreated metastatic colorectal cancer: the PRIME study. J Clin Oncol 28(31): 4697-4705.

Falcone A, Ricci S, Brunetti I, Pfanner E, Allegrini G, Barbara C, Crino L, Benedetti G, Evangelista W, Fanchini L, Cortesi E, Picone V, Vitello S, Chiara S, Granetto C, Porcile G, Fioretto L, Orlandini C, Andreuccetti M, Masi G (2007) Phase III trial of infusional fluorouracil, leucovorin, oxaliplatin, and irinotecan (FOLFOXIRI) compared with infusional fluorouracil, leucovorin, and irinotecan (FOLFIRI) as first-line treatment for metastatic colorectal cancer: the Gruppo Oncologico Nord Ovest. J Clin Oncol 25(13): 1670-1676.

Fornaro L, Lonardi S, Masi G, Loupakis F, Bergamo F, Salvatore L, Cremolini C, Schirripa M, Vivaldi C, Aprile G, Zaniboni A, Bracarda S, Fontanini G, Sensi E, Lupi C, Morvillo M, Zagonel V, Falcone A (2013) FOLFOXIRI in combination with panitumumab as first-line treatment in quadruple wild-type (KRAS, NRAS, HRAS, BRAF) metastatic colorectal cancer patients: a phase II trial by the Gruppo Oncologico Nord Ovest (GONO). Ann Oncol 24(8): 2062-2067.

Gerger A, El-Khoueiry A, Zhang W, Yang D, Singh H, Bohanes P, Ning Y, Winder T, Labonte MJ, Wilson PM, Benhaim L, Paez D, El-Khoueiry R, Absenger G, Lenz HJ (2011) Pharmacogenetic angiogenesis profiling for first-line Bevacizumab plus oxaliplatin-based chemotherapy in patients with metastatic colorectal cancer. Clin Cancer Res 17(17): 5783-5792.

Gruenberger T, Bridgewater J, Chau I, Garcia Alfonso P, Rivoire M, Mudan S, Lasserre S, Hermann F, Waterkamp D, Adam R (2015) Bevacizumab plus mFOLFOX-6 or FOLFOXIRI in patients with initially unresectable liver metastases from colorectal cancer: the OLIVIA multinational randomised phase II trial. Ann Oncol 26(4): 702-708.

Hansen TF, Christensen R, Andersen RF, Garm Spindler KL, Johnsson A, Jakobsen A (2012) The predictive value of single nucleotide polymorphisms in the VEGF system to the efficacy of first-line treatment with bevacizumab plus chemotherapy in patients with metastatic 
colorectal cancer: results from the Nordic ACT trial. Int J Colorectal Dis 27(6): 715-720.

Heinemann V, von Weikersthal LF, Decker T, Kiani A, Vehling-Kaiser U, Al-Batran SE, Heintges T, Lerchenmuller C, Kahl C, Seipelt G, Kullmann F, Stauch M, Scheithauer W, Hielscher J, Scholz M, Muller S, Link H, Niederle N, Rost A, Hoffkes HG, Moehler M, Lindig RU, Modest DP, Rossius L, Kirchner T, Jung A, Stintzing S (2014) FOLFIRI plus cetuximab versus FOLFIRI plus bevacizumab as first-line treatment for patients with metastatic colorectal cancer (FIRE-3): a randomised, open-label, phase 3 trial. Lancet Oncol 15(10): 1065-1075.

Lambrechts D, Lenz HJ, de Haas S, Carmeliet P, Scherer SJ (2013) Markers of response for the antiangiogenic agent bevacizumab. J Clin Oncol 31(9): 1219-1230.

Loupakis F, Cremolini C, Antoniotti C, Lonardi S, Ronzoni M (2015) FOLFOXIRI plus bevacizumab versus FOLFIRI plus bevacizumab as initial treatment for metastatic colorectal cancer (TRIBE study): updated survival results and final molecular subgroups analyses. J Clin Oncol 33(15 Suppl): abstr 3510.

Loupakis F, Cremolini C, Masi G, Lonardi S, Zagonel V, Salvatore L, Cortesi E, Tomasello G, Ronzoni M, Spadi R, Zaniboni A, Tonini G, Buonadonna A, Amoroso D, Chiara S, Carlomagno C, Boni C, Allegrini G, Boni L, Falcone A (2014) Initial therapy with FOLFOXIRI and bevacizumab for metastatic colorectal cancer. N Engl J Med 371(17): 1609-1618.

Loupakis F, Cremolini C, Yang D, Salvatore L, Zhang W, Wakatsuki T, Bohanes P, Schirripa M, Benhaim L, Lonardi S, Antoniotti C, Aprile G, Graziano F, Ruzzo A, Lucchesi S, Ronzoni M, De Vita F, Tonini G, Falcone A, Lenz HJ (2013) Prospective validation of candidate SNPs of VEGF/VEGFR pathway in metastatic colorectal cancer patients treated with first-line FOLFIRI plus bevacizumab. PLoS One 8(7): e66774.

Malvezzi M, Bertuccio P, Levi F, La Vecchia C, Negri E (2014) European cancer mortality predictions for the year 2014. Ann Oncol 25(8): $1650-1656$.

Masi G, Loupakis F, Salvatore L, Fornaro L, Cremolini C, Cupini S, Ciarlo A, Del Monte F, Cortesi E, Amoroso D, Granetto C, Fontanini G, Sensi E, Lupi C, Andreuccetti M, Falcone A (2010) Bevacizumab with FOLFOXIRI (irinotecan, oxaliplatin, fluorouracil, and folinate) as first-line treatment for metastatic colorectal cancer: a phase 2 trial. Lancet Oncol 11(9): $845-852$.

Souglakos J, Androulakis N, Syrigos K, Polyzos A, Ziras N, Athanasiadis A, Kakolyris S, Tsousis S, Kouroussis C, Vamvakas L, Kalykaki A, Samonis G, Mavroudis D, Georgoulias V (2006) FOLFOXIRI (folinic acid, 5-fluorouracil, oxaliplatin and irinotecan) vs FOLFIRI (folinic acid, 5-fluorouracil and irinotecan) as first-line treatment in metastatic colorectal cancer (MCC): a multicentre randomised phase III trial from the Hellenic Oncology Research Group (HORG). Br J Cancer 94(6): 798-805.

Therasse P, Arbuck SG, Eisenhauer EA, Wanders J, Kaplan RS, Rubinstein L, Verweij J, Van Glabbeke M, van Oosterom AT, Christian MC, Gwyther SG (2000) New guidelines to evaluate the response to treatment in solid tumors. European Organization for Research and Treatment of Cancer, National Cancer Institute of the United States, National Cancer Institute of Canada. J Natl Cancer Inst 92(3): 205-216.

Van Cutsem E, Cervantes A, Nordlinger B, Arnold D. on behalf of the EGWG (2014) Metastatic colorectal cancer: ESMO Clinical Practice Guidelines for diagnosis, treatment and follow-updagger. Ann Oncol 25(Suppl 3): iiil-iii9.

Venook A, Niedzwiecki D, Lenz HJ, Innocenti F, Mahoney M (2014) CALGB/SWOG 80405: Phase III trial of irinotecan/5-FU/leucovorin (FOLFIRI) or oxaliplatin/5-FU/leucovorin (mFOLFOX6) with bevacizumab (BV) or cetuximab (CET) for patients (pts) with KRAS wild-type (wt) untreated metastatic adenocarcinoma of the colon or rectum (MCRC). J Clin Oncol 32(5 suppl): abtsr LBA3.

Ychou M, Viret F, Kramar A, Desseigne F, Mitry E, Guimbaud R, Delpero JR, Rivoire M, Quenet F, Portier G, Nordlinger B (2008) Tritherapy with fluorouracil/leucovorin, irinotecan and oxaliplatin (FOLFIRINOX): a phase II study in colorectal cancer patients with non-resectable liver metastases. Cancer Chemother Pharmacol 62(2): 195-201.

This work is published under the standard license to publish agreement. After 12 months the work will become freely available and the license terms will switch to a Creative Commons AttributionNonCommercial-Share Alike 4.0 Unported License.

Supplementary Information accompanies this paper on British Journal of Cancer website (http://www.nature.com/bjc) 